\title{
WEAKLY DENSE IDEALS IN PRIVALOV SPACES OF HOLOMORPHIC FUNCTIONS
}

\author{
Romeo MeŠtrović and Žarko Pavićević
}

\begin{abstract}
In this paper we study the structure of closed weakly dense ideals in Privalov spaces $N^{p}(1<p<\infty)$ of holomorphic functions on the disk $\mathbb{D}:|z|<1$. The space $N^{p}$ with the topology given by Stoll's metric [21] becomes an $F$-algebra. N. Mochizuki [16] proved that a closed ideal in $N^{p}$ is a principal ideal generated by an inner function. Consequently, a closed subspace $E$ of $N^{p}$ is invariant under multiplication by $z$ if and only if it has the form $I N^{p}$ for some inner function $I$. We prove that if $\mathcal{M}$ is a closed ideal in $N^{p}$ that is dense in the weak topology of $N^{p}$, then $\mathcal{M}$ is generated by a singular inner function. On the other hand, if $S_{\mu}$ is a singular inner function whose associated singular measure $\mu$ has the modulus of continuity $O\left(t^{(p-1) / p}\right)$, then we prove that the ideal $S_{\mu} N^{p}$ is weakly dense in $N^{p}$. Consequently, for such singular inner function $S_{\mu}$, the quotient space $N^{p} / S_{\mu} N^{p}$ is an $F$-space with trivial dual, and hence $N^{p}$ does not have the separation property.
\end{abstract}

\section{Introduction and preliminaries}

Let $\mathbb{D}$ denote the unit disk $\{z \in \mathbb{C}:|z|<1\}$ in the complex plane $\mathbb{C}$ and let $\mathbb{T}$ be the boundary of $\mathbb{D}$. For $0<q \leq \infty$ we denote by $L^{q}(\mathbb{T})$ the Lebesgue space with respect to the normalized Lebesgue measure on $\mathbb{T}$. Given $1<p<\infty$, the Privalov class $N^{p}$ consists of all holomorphic functions $f$ on $\mathbb{D}$ such that

$$
\sup _{0 \leq r<1} \int_{0}^{2 \pi}\left(\log ^{+}\left|f\left(r e^{i \theta}\right)\right|\right)^{p} \frac{d \theta}{2 \pi}<+\infty .
$$

These classes were introduced by I. I. Privalov in [17, p. 93], where $N^{p}$ is denoted as $A_{q}$ (with $q=p>1$ ). The study on the spaces $N^{p}$ was continued by Stoll's work [21] in 1977 year. Further, topological and functional properties of these classes were investigated in [1], [5], [6], [12]-[14] and [16]; typically, the notation varied and Privalov was mentioned only in [12] and [14].

Received October 30, 2009; Revised December 29, 2009.

2010 Mathematics Subject Classification. Primary 46J15, 46J20; Secondary 30H05, $30 \mathrm{D} 55$.

Key words and phrases. Privalov space $N^{p}, F$-algebra, weakly dense ideal, singular inner function, topological dual. 
Recall that the Nevanlinna class $N$ consists of all holomorphic functions $f$ on $\mathbb{D}$ such that

$$
\sup _{0 \leq r<1} \int_{0}^{2 \pi} \log ^{+}\left|f\left(r e^{i \theta}\right)\right| \frac{d \theta}{2 \pi}<+\infty .
$$

It is known that for each $f \in N$, the radial limit of $f$ defined as $f\left(e^{i \theta}\right)=$ $\lim _{r \rightarrow 1} f\left(r e^{i \theta}\right)$ exists for almost every $e^{i \theta} \in \mathbb{T}$ (see [7, p. 97]). The Smirnov class $N^{+}$is the set of all functions $f \in N$ such that

$$
\lim _{r \rightarrow 1} \int_{0}^{2 \pi} \log ^{+}\left|f\left(r e^{i \theta}\right)\right| \frac{d \theta}{2 \pi}=\int_{0}^{2 \pi} \log ^{+}\left|f\left(e^{i \theta}\right)\right| \frac{d \theta}{2 \pi} .
$$

For $0<q<\infty$ we denote by $H^{q}$ the classical Hardy space on $\mathbb{D}$, and by $H^{\infty}$ the space of bounded holomorphic function on $\mathbb{D}$. It is known (see [16]) that

$$
N^{s} \subset N^{p}(s>p), \quad \bigcup_{q>0} H^{q} \subset \bigcap_{p>1} N^{p}, \quad \text { and } \bigcup_{p>1} N^{p} \subset N^{+} \subset N,
$$

and these inclusion relations are proper.

Theorem A ([21, Theorem 4.2]). The Privalov space $N^{p}(1<p<\infty)$ (with the notation $\left(\log ^{+} H\right)^{p}$ in [21]) with the topology given by the metric $\rho_{p}$ defined as

$$
\rho_{p}(f, g)=\left(\int_{0}^{2 \pi}\left(\log \left(1+\left|f\left(e^{i \theta}\right)-g\left(e^{i \theta}\right)\right|\right)\right)^{p} \frac{d \theta}{2 \pi}\right)^{1 / p}, \quad f, g \in N^{p},
$$

is an F-algebra, i.e., a complete metrizable topological vector space in which multiplication is continuous.

It is well known $\left[2\right.$, p. 26] that every function $f \in N^{+}$admits a unique factorization of the form

$$
f(z)=B(z) S_{\mu}(z) F(z), \quad z \in \mathbb{D},
$$

where $B$ is the Blaschke product with respect to zeros $\left\{z_{n}\right\} \subset \mathbb{D}$ of $f$ (the set $\left\{z_{n}\right\}$ may be finite), $S_{\mu}$ is a singular inner function, $F$ is an outer function for $N^{+}$, i.e.,

$$
B(z)=z^{m} \prod_{n=1}^{\infty} \frac{\left|z_{n}\right|}{z_{n}} \frac{z_{n}-z}{1-\bar{z}_{n} z}, \quad z \in \mathbb{D},
$$

with $\sum_{n=1}^{\infty}\left(1-\left|z_{n}\right|\right)<\infty, m$ a nonnegative integer,

$$
S_{\mu}(z)=\exp \left(-\int_{0}^{2 \pi} \frac{e^{i t}+z}{e^{i t}-z} d \mu(t)\right)
$$

with positive singular measure $d \mu$, and

$$
F(z)=\lambda \exp \left(\frac{1}{2 \pi} \int_{0}^{2 \pi} \frac{e^{i t}+z}{e^{i t}-z} \log \left|f\left(e^{i t}\right)\right| d t\right),
$$

where $|\lambda|=1$ and $\log \left|f\left(e^{i \theta}\right)\right| \in L^{1}(\mathbb{T})$. 
Recall that a function $I$ of the form

$$
I(z)=B(z) S_{\mu}(z), \quad z \in \mathbb{D},
$$

is called an inner function, and $I$ is a bounded holomorphic function whose boundary values $f\left(e^{i \theta}\right)$ have modulus 1 for almost every $e^{i \theta} \in \mathbb{T}$.

The inner-outer factorization theorem for the classes $N^{p}$ is given by Privalov [17] as follows.

Theorem B ([17, pp. 98-100]; also see [6]). A function $f \in N^{+}$factorized by (1.2) belongs to $N^{p}$ if and only if $\log ^{+}\left|F\left(e^{i \theta}\right)\right| \in L^{p}(\mathbb{T})$.

A closed subspace $E$ of $N^{p}$ is called invariant if $z f \in E$ whenever $f \in E$. Here $z$ denotes the identity function on $\mathbb{D}$. By using a result of Mochizuki [16, Theorem 4], in Section 2 we exhibit the close relationship between inner functions and invariant subspaces of $N^{p}$. We prove (Theorem 2.3) that a closed subspace $E$ of $N^{p}$ is invariant if and only if it has the form $I N^{p}$ for some inner function $I$. Recall that a related result in more general form was proved by Matsugu [12, Theorem 1] using a classical result about invariant subspaces in $L^{2}(\mathbb{T})$ (cf. Remark 3).

For $0<q<\infty$, in Section 3 we consider the space $F^{q}$ consisting of those functions $f$ holomorphic on $\mathbb{D}$ for which

$$
\lim _{r \rightarrow 1}(1-r)^{1 / q} \log ^{+}\left(\max _{|z| \leq r}|f(z)|\right)=0 .
$$

Stoll [21, Theorem 3.2] proved that the space $F^{q}$ with the topology given by the family of seminorms $\left\{\|\cdot\|_{q, c}\right\}_{c>0}$ defined for

$$
f(z)=\sum_{n=0}^{\infty} a_{n} z^{n} \in F^{q}
$$

as

$$
\|f\|_{q, c}=\sum_{n=0}^{\infty}\left|a_{n}\right| \exp \left(-c n^{1 /(q+1)}\right)<\infty,
$$

is a countably normed Fréchet algebra. By a result of Eoff [5, Theorem 4.2], $F^{p}$ is the Fréchet envelope of $N^{p}$, and hence $F^{p}$ and $N^{p}$ have the same topological duals. Thus, the family of seminorms $\left\{\|\cdot\|_{p, c}\right\}_{c>0}$ induces on $N^{p}$ the strongest locally convex (metrizable) topology that is weaker than the metric $\rho_{p}$ topology.

In Section 4 we characterize the weak closure $[E]_{w}$ of a vector subspace $E$ of $N^{p}$, that is, the closure with respect to the weak topology defined on $N^{p}$ in the usual way. The non locally convex phenomenon of interest here is the existence of proper, closed subspaces of $N^{p}$ that are dense in the weak topology. We show that for any inner function $I$, the weak closure of an invariant subspace $I N^{p}$ of $N^{p}$ is again an invariant subspace. Hence, $\left[I N^{p}\right]_{w}=I_{w} N^{p}$ for some inner function $I_{w}$. We shall say that $I$ is a weak outer function in $N^{p}$ if $I N^{p}$ is weakly dense in $N^{p}$; that is, if $I_{w} \equiv 1$. We prove (Corollary 4.7) that an inner function 
$I$ is weak outer in $N^{p}$ if and only if the space $I N^{p}$ is dense in $F^{p}$, that is, if and only if the space $I F^{p}$ is dense in $F^{p}$.

A closed subspace $E$ of $N^{p}$ will be said to have the separation property if each $f \in N^{p}$ that is not in $E$ can be separated from $E$ by a continuous linear functional on $N^{p}$; that is, if there exists a continuous linear functional $\varphi$ on $N^{p}$ such that $\varphi(E)=0$ but $\varphi(f) \neq 0$. We will say that $E$ has the HahnBanach property if each continuous linear functional on $E$ can be extended to a continuous linear functional on the whole space $N^{p}$. In Section 4 (Theorem 4.10) we prove that if $I$ is any Blaschke product, then $I N^{p}$ has the separation property. In the case if $I$ is a finite Blaschke product, we prove that $I N^{p}$ has the Hahn-Banach property.

It was proved in [4, Theorem 14] that if $S$ is a nontrivial singular inner function whose associated singular measure has the modulus of continuity $O\left(t \log \frac{1}{t}\right)$, then $I$ is a weak outer function in any space $H^{q}$ with $0<q<1$. In particular, it is not known whether an inner function can be weak outer for some values of $0<p<1$, but not for others. In our main result given in Section 5 (Theorem 5.5), for any fixed $1<p<\infty$ we present a large class of positive singular measures $\mu$, depending on $p$, such that associated singular inner functions are weak outer functions in the Privalov space $N^{p}$. More precisely, Theorem 5.5 asserts that if $S_{\mu}$ is a non-trivial singular inner function with the associated measure $\mu$ whose modulus of continuity $\omega_{\mu}(t)=O\left(t^{(p-1) / p}\right)$, then the ideal $S_{\mu} N^{p}$ is weakly dense in $N^{p}$. Consequently, such a singular inner function $S_{\mu}$ is a weak outer function in $N^{p}$ and the quotient space $N^{p} / S_{\mu} N^{p}$ is an $F$-space with trivial dual (Corollaries 5.6 and 5.7).

Recall that it was shown in [19, Proposition 4] that a closed ideal in $N^{+}$is weakly dense if and only if it is generated by a singular inner function $S_{\mu}$ with $\mu$ a continuous singular measure.

Remark 1. The above notions and definitions are motivated by the famous Beurling's theorem (see [9, Ch. 7, p. 99], [8, Lecture II]) and results from [4] related to the linear space structure of the Hardy space $H^{q}$ with $0<q<1$. Beurling's invariant subspace theorem to $H^{q}$, tells that there is a one-to-one correspondence between inner functions and invariant subspaces; each invariant subspace of $H^{q}$ being of the form $I H^{q}$, where $I$ is an inner function. Beurling's theorem can also be viewed as a result on approximation. In this formulation it states that the polynomial multiples of an $H^{q}$ function form a dense subset of $H^{q}$ if and only if that function is outer (see [2, Section 7.3]).

In [4] Duren, Romberg, and Shields added a new dimension to Beurling's theorem by proving that when $0<q<1$ some inner functions (not identically 1) give rise to weakly dense invariant subspaces of $H^{q}$. In view of the approximation-theoretic formulation of Beurling's theorem, Duren, Romberg, and Shields called such inner functions weakly outer. 


\section{Invariant subspaces of $N^{p}$}

Denote by $\mathcal{P}$ the space of all polynomials. Let $X$ be a topological vector space of holomorphic functions on $\mathbb{D}$ so that $H^{\infty} \subset X$ and convergence in $X$ implies uniform convergence on compact subsets of $\mathbb{D}$. Suppose that $1 \in X$ and that $f \in X$ implies $P f \in X$ for every polynomial $P \in \mathcal{P}$. If $f \in X$, then $\operatorname{cl}(\mathcal{P} f)$ denotes the closure of $\mathcal{P} f:=\{P f: P \in \mathcal{P}\}$; so $\operatorname{cl}(\mathcal{P} f)$ is the smallest invariant (under multiplication by $z$ ) closed subspace containing $f$. Recall that an invariant subspace of $X$ is defined as a closed subspace $E$ of $X$ such that $z f \in E$ whenever $f \in E$. A function $f \in X$ is said to be cyclic in $X$ if $\operatorname{cl}(\mathcal{P} f)=X$. This is equivalent to the fact that there exists a sequence $\left\{P_{n}\right\}_{n}$ of polynomials such that $P_{n} f \rightarrow 1$ as $n \rightarrow \infty$ in the topology of $X$. Thus, a function $f \in X$ is cyclic if it generates $X$ as an invariant subspace, that is, the smallest invariant subspace of $X$ containing the function is the whole space.

By Beurling's theorem mentioned previously, a function in $H^{q}(0<q<\infty)$ is cyclic if and only if it is outer. Using the following result of Mochizuki, we can easily obtain the analogous characterization of cyclic functions in $N^{p}$.

Theorem 2.1 ([16, Theorem 4]). Let $\mathcal{M}$ be a closed ideal in $N^{p}$ which is not identically 0 . Then there is a unique (modulo constants) inner function I such that $\mathcal{M}=I N^{p}$.

Lemma 2.2. A closed subspace $E$ of $N^{p}$ is invariant if and only if it is an ideal.

Proof. The proof is routine, by using the fact that $N^{p}$ is a topological algebra in which polynomials are dense (see [13, the proof of the assertion 2.3]), and hence may be omitted.

As an immediate consequence of Theorem 2.1 and Lemma 2.2, we obtain the following $N^{p}$-analogue of Beurling's theorem on invariant subspaces of the Hardy space.

Theorem 2.3. A closed subspace $E$ of $N^{p}$ is invariant if and only if it has the form $I N^{p}$ for some inner function $I$.

Remark 2. By [19, Theorem 2] and Lemma 2.2 with $N^{+}$instead of $N^{p}$, it follows that Theorem 2.3 is also true for the Smirnov class $N^{+}$.

Theorem 2.3 shows that there is a one-to-one correspondence between inner functions and invariant subspaces of $N^{p}$; so each invariant subspace of $N^{p}$ being of the form $I N^{p}$, where $I$ is an inner function.

The approximative version of Theorem 2.3 is given as follows.

Theorem 2.4 ([13, the assertion 2.3 on p. 99]). Let $f \in N^{p}$ with the factorization $f=B S F=I F$ given by Theorem $B$, and let $\mathcal{P}$ denote the space of all polynomials over $\mathbb{C}$. Then the set $B S N^{p}=I N^{p}=\left\{I g: g \in N^{p}\right\}$ becomes the closure of $\mathcal{P} f:=\{P f: P \in \mathcal{P}\}$ in $N^{p}$ with respect to the metric topology $\rho_{p}$. 
Clearly, Theorem 2.4 can be formulated in terms of cyclic functions in $N^{p}$ as follows.

Theorem 2.5. A function $f \in N^{p}$ is cyclic in $N^{p}$ if and only if $f$ is an outer function.

Remark 3. In [12] Matsugu characterized invariant subspaces of the space $\left(\log ^{+}\right)^{p}(\mathbb{T})(1 \leq p<\infty)$ consisting of all measurable functions $f$ on the circle $\mathbb{T}$ such that

$$
\|f\|:=\int_{0}^{2 \pi}\left(\left(\log \left(1+\left|f\left(e^{i \theta}\right)\right|\right)\right)^{p} \frac{d \theta}{2 \pi}\right)^{1 / p}<+\infty .
$$

As noticed in [12], it can be easily shown that $\left(\log ^{+}\right)^{p}(\mathbb{T})$ is an $F$-space with respect to the metric $\tilde{\rho}_{p}(f, g)=\|f-g\|, f, g \in\left(\log ^{+}\right)^{p}(\mathbb{T})$. Furthermore, it follows by the Riesz Uniqueness Theorem that the Privalov space $N^{p}$ can be identified with the space $N_{*}^{p}$ of all boundary functions of $N^{p}$, i.e.,

$$
N_{*}^{p}=\left\{f^{*}: f \in N^{p}\right\} .
$$

Then $N_{*}^{p}\left(\equiv N^{p}\right)$ is a closed subspace of $\left(\log ^{+}\right)^{p}(\mathbb{T})$ (cf. [23, Theorem 1] for $p=1$ and [21, Theorem 4.2] for $p>1$ ). By using the classical result about invariant subspaces in $L^{2}(\mathbb{T})([8$, Theorems 2 and 3]; [12, Theorem A]), Matsugu $\left[12\right.$, Theorems 1 and 2] described the set of all invariant subspaces of $\left(\log ^{+}\right)^{p}(\mathbb{T})$. These results immediately yield our Theorem 2.3 (extended for $p=1$ with $\left.N^{1} \equiv N^{+}\right)$.

\section{Fréchet envelope and topological dual of $N^{p}$}

In connection with the spaces $N^{p}(1<p<\infty)$, Stoll in [21] also studied the spaces $F^{q}(0<q<\infty)$ with the notation $F_{1 / q}$ in [21], consisting of those functions $f$ holomorphic on $\mathbb{D}$ for which

$$
\lim _{r \rightarrow 1}(1-r)^{1 / q} \log ^{+} M_{\infty}(r, f)=0,
$$

where

$$
M_{\infty}(r, f)=\max _{|z| \leq r}|f(z)| .
$$

Here, as always in the sequel, we will need some Stoll's results concerning the spaces $F^{q}$ only with $1<q<\infty$, and hence we will assume that $q=p>1$ be any fixed number.

Theorem 3.1 ([21, Theorem 2.2]). Suppose that $f(z)=\sum_{n=0}^{\infty} a_{n} z^{n}$ is a holomorphic function on $\mathbb{D}$. Then the following statements are equivalent.

(a) $f \in F^{p}$.

(b) There exists a sequence $\left\{c_{n}\right\}_{n}$ of positive real numbers with $c_{n} \rightarrow 0$ such that

$$
\left|a_{n}\right| \leq \exp \left(c_{n} n^{1 /(p+1)}\right), \quad n=0,1,2, \ldots
$$


(c) For any $c>0$,

$$
\|f\|_{p, c}:=\sum_{n=0}^{\infty}\left|a_{n}\right| \exp \left(-c n^{1 /(p+1)}\right)<\infty .
$$

Note that in view of Theorem $3.1((\mathrm{a}) \Leftrightarrow(\mathrm{c}))$, it is defined by (3.1) the family of seminorms $\left\{\|\cdot\|_{p, c}\right\}_{c>0}$ on $F^{p}$.

Moreover, Stoll defined the family of seminorms $\left\{|\|\cdot\||_{p, c}\right\}_{c>0}$ on $F^{p}$ given as

$$
\|\| f \|_{p, c}=\int_{0}^{1} \exp \left(-c(1-r)^{-1 / p}\right) M_{p}(r, f) d r, \quad f \in F^{p}
$$

where

$$
M_{p}(r, f)=\left(\int_{0}^{2 \pi}\left|f\left(r e^{i \theta}\right)\right|^{p} \frac{d \theta}{2 \pi}\right)^{1 / p} .
$$

By [21, Proposition 3.1], $\left\{\|\cdot\|_{p, c}\right\}_{c>0}$ and $\left\{|\|\cdot\||_{p, c}\right\}_{c>0}$ are equivalent families of seminorms. More precisely, Stoll proved the following result.

Theorem 3.2 ([21, Proposition 3.1]). For each $c>0$, there is a constant $A$ depending only on $p$ and $c$, such that

$$
\begin{aligned}
& \qquad\|f\|_{p, c} \leq\|f\|_{p, c_{1}} \text { and }\|f\|_{p, c} \leq A\|f\| \|_{p, c_{2}}, \\
& \text { with } c_{1}=c^{p /(p+1)} \text { and } c_{2}=\left(\frac{c}{12}\right)^{p /(p+1)} \text {. }
\end{aligned}
$$

Recall that a locally convex $F$-space is called a Fréchet space, and a Fréchet algebra is a Fréchet space that is an algebra in which multiplication is continuous.

Theorem 3.3 ([21, Theorem 3.2]). The space $F^{p}$ with the topology given by the family of seminorms $\left\{\|\cdot\|_{p, c}\right\}_{c>0}$, or $\left\{\mid\|\cdot\| \|_{p, c}\right\}_{c>0}$, is a countably normed Fréchet algebra. Moreover,

$$
\|f g\|_{p, c} \leq\|f\|_{p, c^{\prime}}\|g\|_{p, c^{\prime}} \quad \text { for all } \quad f, g \in F^{p}
$$

where $c^{\prime}=c \cdot 2^{-p /(p+1)}$. Furthermore, if $f \in F^{p}$, then $f_{r} \rightarrow f$ as $r \rightarrow 1$ in the topology of $F^{p}$ where $f_{r}(z)=f(r z)$ with $z \in \mathbb{D}$ and $0<r<1$.

For our purposes the most important connection between spaces $N^{p}$ and $F^{p}$ is given by the following result.

Theorem 3.4 ([21, Theorem 4.3]). For any fixed $p>1$ the following assertions hold.

(a) $N^{p}$ is a dense subspace of $F^{p}$.

(b) The topology on $F^{p}$ defined by the family of seminorms (3.1) or (3.2) is weaker than the topology on $N^{p}$ given by the metric $\rho_{p}$ defined by (1.1). 
(c) For each $q>p$ there exists a function $f_{q} \in N^{p}$ such that

$$
\limsup _{r \rightarrow 1}(1-r)^{1 / q} \log ^{+} M_{\infty}\left(r, f_{q}\right)>0,
$$

i.e., $N^{p}$ is not contained in $F^{q}$ for none $q>p$.

Remark 4. Recall that the spaces $F^{p}$ have also been studied independently by Zayed ([25] and [26]); many of the results in [24] parallel those of Stoll in [21], albeit in a more general setting. For $p=1$, the space $F_{1}$ has been denoted by $F^{+}$and has been studied by Yanagihara in [24] and [23]. It was shown in [24] and [23] that $F^{+}$is actually the containing Fréchet space for $N^{+}$, i.e., $N^{+}$with the initial topology embeds densely into $F^{+}$, under the natural inclusion, and $F^{+}$and $N^{+}$have the same topological duals.

Observe that the space $F^{p}$ topologised by the family of seminorms $\left\{\|\cdot\|_{p, c}\right\}_{c>0}$ given by (3.1) is metrizable by the metric $d_{p}$ defined as

$$
d_{p}(f, g)=\sum_{n=1}^{\infty} 2^{-n} \frac{\|f-g\|_{p, 1 / n^{p /(p+1)}}}{1+\|f-g\|_{p, 1 / n^{p /(p+1)}}}, \quad f, g \in F^{p} .
$$

The following result describes the topological dual of the space $\left(F^{p}, d_{p}\right)$.

Theorem 3.5 ([21, Theorem 3.3]). If $\gamma$ is a continuous linear functional on $F^{p}$, then there exists a sequence $\left\{\gamma_{n}\right\}_{n}$ of complex numbers with $\gamma_{n}=$ $O\left(\exp \left(-c n^{1 /(p+1)}\right)\right)$ for some $c>0$, such that

$$
\gamma(f)=\sum_{n=0}^{\infty} a_{n} \gamma_{n}
$$

where $f(z)=\sum_{n=0}^{\infty} a_{n} z^{n} \in F^{p}$, with convergence being absolute. Conversely, if $\left\{\gamma_{n}\right\}$ is a sequence of complex numbers for which

$$
\gamma_{n}=O\left(\exp \left(-c n^{1 /(p+1)}\right)\right),
$$

then (3.4) defines a continuous linear functional on $F^{p}$.

Let us recall that if $X=(X, \tau)$ is an $F$-space whose topological dual (the set of all continuous linear functionals on $X) X^{*}$ separates the points of $X$, then its Fréchet envelope $\hat{X}$ is defined to be the completion of the space $\left(X, \tau^{c}\right)$, where $\tau^{c}$ is the strongest locally convex (necessarily metrizable) topology on $X$ that is weaker than $\tau$. In fact, it is known that $\tau^{c}$ is equal to the Mackey topology of the dual pair $\left(X, X^{*}\right)$, i.e., to the unique maximal locally convex topology on $X$ for which $X$ still has dual space $X^{*}$ (see [20, Theorem 1]). For each metrizable locally convex topology $\tau$ on $X,(X, \tau)$ is a Mackey space, i.e., $\tau$ coincides with the Mackey topology of the dual pair $\left(X, X^{*}\right)$ (see [10, Corollary 22.3, p. 210]).

Eoff ([5, the proof of Theorem 4.2]) showed that the topology of $F^{p}, p>1$, (resp., $F^{1}=F^{+}$) is stronger than that of the Fréchet envelope of $N^{p}$ (resp., $N^{+}$). As an immediate consequence of this result, we obtain the following statements. 
Theorem 3.6 ([5, Theorem 4.2, the case $p>1])$. For each $p>1, F^{p}$ is the Fréchet envelope of $N^{p}$.

Theorem 3.7 ([14, Theorem 2]). The spaces $N^{p}$ and $F^{p}$ have the same dual spaces in the sense that every continuous linear functional on $F^{p}$ (given by (3.4)) restricts to one on $N^{p}$, and every continuous linear functional on $N^{p}$ extends continuously to one on $F^{p}$.

Remark 5. Theorem 3.7 is proved in [14] directly, by using the characterization of multipliers from $N^{q}$ into $H^{\infty}([14$, Theorem 1]). The dual space of the Smirnov class $N^{+}$is completely described by Yanagihara in [23] and McCarthy in $[15]$.

The following result establishes the fact that the dual of $N^{p}$ contains many elements.

Theorem 3.8. For any fixed $\xi \in \mathbb{D}$ and all $k=0,1,2 \ldots$, the functional $\delta_{\xi}^{(k)}$ defined as $\delta_{\xi}^{(k)}(f)=f^{(k)}(\xi), f \in N^{p}$, is a continuous linear functional on $N^{p}$. In particular, for $k=0$ every point evaluation $\delta_{\xi}$ defined as $\delta_{\xi}(f)=f(\xi)$, $f \in N^{p}$, is a multiplicative continuous linear functional on $N^{p}$.

Proof. It is easy to verify that for a fixed $\xi \in \mathbb{D}$, the sequence $\left\{\gamma_{n}\right\}_{n}:=\left\{\xi^{n}\right\}_{n}$ satisfies the condition from Theorem 3.5 for any $p>1$, and hence it generates by (3.4) the continuous linear functional $\gamma$ such that $\gamma(f)=f(\xi)=\delta_{\xi}(f)$ for $f \in F^{p}$. Obviously, $\delta_{\xi}$ is multiplicative.

Similarly, for fixed $\xi \in \mathbb{D}$ and $k \in \mathbb{N}$, the sequence $\left\{\left(\xi^{n}\right)^{(k)}\right\}_{n}$ with terms

$$
\left(\xi^{n}\right)^{(k)}= \begin{cases}n(n-1) \cdots(n-k+1) \xi^{n-k} & \text { for } n \geq k \\ 0 & \text { for } n<k\end{cases}
$$

also satisfies the growth estimate from Theorem 3.5 for any $p>1$, and thus by Theorem 3.7, every functional $\delta_{\xi}^{(k)}=f^{(k)}(\xi)$ is a continuous linear functional on $N^{p}$.

\section{Invariant subspaces and linear functionals on $N^{p}$}

Recall that all the notions, statements and their proofs in this section are analogous to those for the spaces $H^{q}$ with $0<q<1$ given in [4, Section 6].

By Theorem 2.3, each invariant subspace of $N^{p}$ being of the form $I N^{p}$, where $I$ is an inner function. Thus there is a one-to-one correspondence between inner functions and invariant subspaces of $N^{p}$. Since $N^{p}$ is not locally convex, it is possible that some closed ideals are weakly dense.

Recall that we may introduce the weak topology on $N^{p}$ in the usual way. The basic weak neighborhoods of zero are defined by

$$
V\left(\varphi_{1}, \ldots, \varphi_{n} ; \varepsilon\right)=\left\{f \in N^{p}:\left|\varphi_{i}(f)\right|<\varepsilon, i=1, \ldots, n\right\},
$$

where $\varepsilon>0$ and $n \in \mathbb{N}$ are arbitrary, and $\varphi_{1}, \ldots, \varphi_{n}$ are arbitrary continuous linear functionals on $N^{p}$. 
For a subset $E$ of $N^{p}$, denote by $[E]$ the closure of $E$ in $N^{p}$, by $[E]_{w}$ the closure of $E$ in the weak topology of $N^{p}$, and by $[E]_{F^{p}}$ the closure of $E$ in $F^{p}$ with respect to the topology of $F^{p}$ given by the family of seminorms (3.1) or (3.2). Denote by $\left(N^{p}\right)^{*}$ the topological dual of $N^{p}$, i.e., the set of all continuous linear functional on $N^{p}$ with respect to the metric topology $\rho_{p}$. It is easy to check that a weakly closed subset of $N^{p}$ is also closed in $N^{p}$ with respect to the metric topology $\rho_{p}$; that is, the weak topology is weaker than the initial $N^{p}$ topology. The weak topology is always locally convex. Clearly, by Theorem $3.8, N^{p}$ has the point separation property. This means that for every function $f \in N^{p}$ which is not identically equal to zero, there is a non-trivial continuous linear functional $\varphi$ on $N^{p}$ such that $\varphi(f) \neq 0$, and hence the weak topology is also Hausdorff.

For vector subspaces of $N^{p}$ there is another description of the weak closure that we shall use (see [4, p. 54]). The weak topology of $N^{p}$ is locally convex, and hence by [10, p. 154, Corollary 17.3], a linear functional on $N^{p}$ is weakly continuous if and only if it is continuous with respect to the metric topology $d_{p}$ given by (3.3). Thus $[E]_{w}$ consists of all those functions $f \in N^{p}$ which cannot be separated from $E$ by a linear functional in $\left(N^{p}\right)^{*}$. Namely, by [10, p. 154, 17.1], in any locally convex topological vector space a convex set $E$ is closed if and only if it is weakly closed, or equivalently, if and only if each point not in it can be separated from it by a linear functional. In the case when $E$ is a vector subspace, this becomes: $f \in[E]_{w}$ if and only if $\phi(f)=0$ for every functional $\phi \in E^{\perp}$, where $E^{\perp}$ is the set of all $\phi \in\left(N^{p}\right)^{*}$ which vanish on $E$. Thus we have the following result.

Lemma 4.1. A closed subspace of $N^{p}$ has the separation property if and only if it is weakly closed.

Lemma 4.2. The weak closure of any invariant subspace $I N^{p}$ of $N^{p}$ is again an invariant subspace.

Proof. We follow the proof of Lemma 7 in [4, pp. 54-55]. Suppose that $f \in$ $\left[I N^{p}\right]_{w}$, where $I$ is an inner function. We must prove that a function $z f(z)$ is in the weak closure of $I N^{p}$. According to the argument preceding Lemma 4.1, this is equivalent to the fact that $\phi(z f)=0$ for each $\phi \in\left(I N^{p}\right)^{\perp}$. Now define the linear functional $\phi_{1}$ on $N^{p}$ as

$$
\phi_{1}(f)=\phi(z f), \quad f \in N^{p} .
$$

Since $\phi_{1}(I g)=\phi(I z g)=0$ for each $g \in N^{p}$, we see that $\phi_{1} \in\left(I N^{p}\right)^{\perp}$. From this and the assumption $f \in\left[I N^{p}\right]_{w}$ it follows that $\phi_{1}(f)=0$, and hence $\phi(z f)=0$. This concludes the proof.

Theorem 4.3. For any inner function I there is a unique (modulo constants) inner function $I_{w}$ such that

$$
\left[I N^{p}\right]_{w}=I_{w} N^{p}
$$

Furthermore, $J=I / I_{w}$ is again an inner function. 
Proof. By Lemma 4.2, $\left[I N^{p}\right]_{w}$ is an invariant subspace of $I N^{p}$ for any inner function $I$. Thus by Theorem 2.3, there is an inner function $I_{w}$ for which

$$
\left[I N^{p}\right]_{w}=I_{w} N^{p} .
$$

The uniqueness (modulo constants) of a function $I_{w}$ follows immediately from the uniqueness of the factorization of $N^{p}$ functions (Theorem B). Finally, since the weak topology is weaker than the metric topology $\rho_{p}$ on $N^{p}$, we conclude that $I N^{p}=\left[I N^{p}\right] \subseteq\left[I N^{p}\right]_{w}$. Therefore, it follows by (4.1) that $I N^{p} \subseteq I_{w} N^{p}$, and hence $J=I / I_{w}$ is an inner function. This completes the proof.

Lemma 4.4. If $E$ is a vector subspace of $N^{p}$, then

$$
[E]_{w}=[E]_{F^{p}} \cap N^{p} .
$$

Furthermore, $[E] \subseteq[E]_{w}$ and $[E]_{w}$ is closed in $N^{p}$.

Proof. As noticed above, $[E]_{w}$ consists of all those functions $f \in N^{p}$ that cannot be separated from $E$ by a linear functional on the dual space $\left(N^{p}\right)^{*}$ of $N^{p}$. On the other hand, by Theorem 3.7, the spaces $N^{p}$ and $F^{p}$ have the same dual spaces, and hence $[E]_{F^{p}} \cap N^{p}$ consists of all those $f \in N^{p}$ that cannot be separated from it by a linear functional. Therefore, $[E]_{w}=[E]_{F^{p}} \cap N^{p}$. Further, $[E] \subseteq[E]_{w}$ is immediate from the fact that the weak topology is weaker than the metric topology $\rho_{p}$ on $N^{p}$. Finally, it remains to show that $[E]_{w}$ is closed in $N^{p}$. Suppose $\left\{f_{n}\right\}$ is a sequence in $[E]_{w}$ such that $f_{n} \rightarrow f$ in $N^{p}$ as $n \rightarrow \infty$ for some $f \in N^{p}$. We must show that $f \in[E]_{w}$. As $\varphi\left(f_{n}\right) \rightarrow \varphi(f)$ as $n \rightarrow \infty$ for each $\varphi \in\left(N^{p}\right)^{*}$, this means that $f_{n} \rightarrow f$ weakly in $N^{p}$ as $n \rightarrow \infty$. Hence, $f \in\left[[E]_{w}\right]_{w}=[E]_{w}$, as desired.

Corollary 4.5. If $\mathcal{M}$ is an ideal in $N^{p}$, then both $[\mathcal{M}]$ and $[\mathcal{M}]_{w}$ are also ideals in $N^{p}$.

Proof. Clearly, since the multiplication is continuous in $N^{p}$, if $\mathcal{M}$ is an ideal in $N^{p}$, so is $[\mathcal{M}]$. Moreover, since the multiplication is also continuous in $F^{p}$ and by Theorem 3.4(a), $N^{p}$ is a dense subspace of $F^{p}$, it follows that $[\mathcal{M}]_{F^{p}}$ is an ideal in $F^{p}$. This fact together with (4.2) yields that $[\mathcal{M}]_{w}$ is an ideal in $N^{p}$.

Although the weak topology of $N^{p}$ is not metrizable, the following corollary shows that, for vector subspaces of $N^{p}$ the weak closure can be formed by adjoining limits of sequences.

Corollary 4.6. If $E$ is a vector subspace of $N^{p}$, then each function in the weak closure of $E$ is the weak limit of a sequence of elements of $E$.

Proof. Assume $f \in[E]_{w}$. By Lemma 4.4, $f \in[E]_{F^{p}}$ and so there is a sequence $\left\{f_{n}\right\}_{n}$ in $E$ such that $f_{n} \rightarrow f$ in the topology of $F^{p}$. Hence $f_{n} \rightarrow f$ weakly in $F^{p}$, which is the same thing as $f_{n} \rightarrow f$ weakly in $N^{p}$.

Corollary 4.7. Let I be an inner function. The following three statements are equivalent. 
(i) $\left[I N^{p}\right]_{w}=N^{p}$.

(ii) $I N^{p}$ is dense in $F^{p}$.

(iii) $I F^{p}$ is dense in $F^{p}$.

Proof. (i) $\Leftrightarrow$ (ii) follows immediately from (4.2) of Lemma 4.4 and the fact that $N^{p}$ is a dense subspace of $F^{p}$.

(ii) $\Rightarrow$ (iii) is obvious, in view of the fact that $I N^{p} \subset I F^{p}$.

(iii) $\Rightarrow$ (ii). If $f \in F^{p}$ is arbitrary, then by (iii) there is a sequence $\left\{f_{n}\right\}_{n}$ in $F^{p}$ such that $I f_{n} \rightarrow f$ in $F^{p}$. Since $N^{p}$ is a dense subspace of $F^{p}$, there exists a sequence $\left\{g_{n}\right\}_{n}$ in $N^{p}$ such that $f_{n}-g_{n} \rightarrow 0$ in $F^{p}$. From this fact and the continuity of multiplication in $F^{p}$, it follows that $I f_{n}-I g_{n} \rightarrow 0$ in $F^{p}$, and hence $I g_{n} \rightarrow f$ in $F^{p}$. Thus $f$ belongs to $\left[I N^{p}\right]_{F^{p}}$, and so (ii) is satisfied.

If $T$ is a continuous linear operator on $N^{p}$, then the adjoint operator $T^{*}$ on $\left(N^{p}\right)^{*}$ is defined by the usual formula

$$
\left(T^{*} \varphi\right)(f)=\varphi(T f), \quad \varphi \in\left(N^{p}\right)^{*}, f \in N^{p} .
$$

Lemma 4.8. Every continuous linear operator $T$ on $N^{p}$ is weakly continuous.

Proof. Assume $f_{\alpha} \rightarrow f$ weakly, that is, $\psi\left(f_{\alpha}\right) \rightarrow \psi(f)$ for all $\psi \in\left(N^{p}\right)^{*}$. Then we have

$$
\psi\left(T f_{\alpha}\right)=\left(T^{*} \psi\right)\left(f_{\alpha}\right) \rightarrow\left(T^{*} \psi\right)(f)=\psi(T f),
$$

whence it follows that $T f_{\alpha} \rightarrow T f$ weakly, as desired.

Lemma 4.9. Let $I$ and $J$ be arbitrary inner functions. Then

$$
\left[I_{w} J N^{p}\right] \subseteq\left[I J N^{p}\right]_{w},
$$

where $I_{w}$ is the inner function such that $\left[I N^{p}\right]_{w}=I_{w} N^{p}$.

Proof. For a fixed function $g \in N^{p}$, define the linear operator $T_{g}$ on $N^{p}$ as

$$
T_{g}(f)=J g f, \quad f \in N^{p} .
$$

Since the multiplication in $N^{p}$ is continuous, it follows that $T_{g}$ is a continuous linear operator, and hence by Lemma $4.8, T_{g}$ is weakly continuous. Therefore, if we choose a sequence $\left\{f_{n}\right\}_{n}$ in $N^{p}$ such that $I f_{n} \rightarrow I_{w}$ weakly, then $T_{g}\left(I f_{n}\right) \rightarrow$ $T_{g}\left(I_{w}\right)$ weakly, i.e., $I J\left(g f_{n}\right) \rightarrow J I_{w} g$ weakly. Hence, $J I_{w} g \in\left[I J N^{p}\right]_{w}$, which implies $I_{w} J N^{p} \subseteq\left[I J N^{p}\right]_{w}$. Therefore, in view of the fact that by Lemma 4.4, $\left[\left[I J N^{p}\right]_{w}\right]=\left[I J N^{p}\right]_{w}$, we obtain

$$
\left[I_{w} J N^{p}\right] \subseteq\left[\left[I J N^{p}\right]_{w}\right]=\left[I J N^{p}\right]_{w} .
$$

This yields a desired inclusion.

Recall that a closed subspace $E$ of $N^{p}$ will be said to have the separation property if each function $f \in N^{p}$ that is not in $E$ can be separated from $E$ by a continuous linear functional on $N^{p}$; that is, if there exists a continuous linear functional $\varphi$ on $N^{p}$ such that $\varphi(E)=0$ but $\varphi(f) \neq 0$. We will say that $E$ has the Hahn-Banach property if each continuous linear functional on $E$ can be extended to a continuous linear functional on the whole space $N^{p}$ (we do not 
require that the norm of the functional be preserved in the extension). We are now ready to state the following result.

Theorem 4.10. Let I be an inner function. Then the following assertions hold.

(i) If $I$ is a finite Blaschke product, then $I N^{p}$ has both the separation property and the Hahn-Banach property.

(ii) If I is any Blaschke product, then $I N^{p}$ has the separation property.

(iii) If the space $I N^{p}$ does not have the separation property and if $J$ is any inner function, then also IJN $N^{p}$ does not have the separation property.

Proof. (i) If $I$ is a finite Blaschke product, then the space $I N^{p}$ has a finite co-dimension. Hence, the assertion (i) follows immediately from the fact that in any topological vector space a closed subspace of finite co-dimension has both the separation and the Hahn-Banach properties.

(ii) If $I$ is a Blaschke product and if $h \notin I N^{p}$, then either $h$ does not vanish at one of the zeros of $I$ or $h$ has a zero of lower multiplicity. In the first case, let $\xi \in \mathbb{D}$ be a zero of $I$ such that $h(\xi) \neq 0$. For such a $\xi$ consider the point evaluation $\delta_{\xi}$ on $N^{p}$ defined as $\delta_{\xi}(f)=f(\xi), f \in N^{p}$. Then by Theorem $3.8, \delta_{\xi}$ is a continuous linear functional on $N^{p}$. Obviously, $\delta_{\xi}(g)=0$ for all $g \in I N^{p}$ but $\delta_{\xi}(h)=h(\xi) \neq 0$, and hence $\delta_{\xi}$ is a desired separating linear functional. In the second case, suppose that $\xi \in \mathbb{D}$ is a zero of $I$ of the multiplicity $k \geq 1$, and hence of the multiplicity less than $k$ for $h$, that is, $h^{(k)}(\xi) \neq 0$. Now consider a linear functional on $N^{p}$ by $\delta_{\xi}^{(k)}(f)=f^{(k)}(\xi), f \in N^{p}$. By Theorem 3.8, $\delta_{\xi}^{(k)}$ is also a continuous linear functional on $N^{p}$. Clearly, $\delta_{\xi}^{(k)}(g)=0$ for all $g \in I N^{p}$ while $\delta_{\xi}^{(k)}(h)=h^{(k)}(\xi) \neq 0$, and thus $\delta_{\xi}^{(k)}$ is a desired separating linear functional.

(iii) Suppose that $I J N^{p}$ has the separation property for some inner function $J$, and so by Lemma 4.1,

$$
\left[I J N^{p}\right]_{w}=I J N^{p} .
$$

By the assumption, the space $I N^{p}$ does not have the separation property, and hence by Lemma 4.1 , it is not weakly closed. This means that $I \neq I_{w}$ (modulo constants), where $I_{w}$ is an inner function associated to $I$ (see Theorem 4.3) such that

$$
\left[I N^{p}\right]_{w}=I_{w} N^{p} .
$$

Since $I N^{p}$ is a weakly dense subspace of $I_{w} N^{p}$, by (4.3) and Lemma 4.9, we have

$$
I J N^{p}=\left[I J N^{p}\right]_{w} \supseteq\left[I_{w} J N^{p}\right]_{w} \supseteq I_{w} J N^{p} \supseteq I J N^{p} .
$$

From the above inclusions we see that must be $I_{w} J N^{p}=I J N^{p}$. Thus, $I_{w} J$ is in $I J N^{p}$, and hence there is an inner function $I_{1}$ such that $I_{w} J=I J I_{1}$. It follows that $I_{w}=I I_{1}$, and hence $I_{w} / I=I_{1}$ is an inner function. On the other hand, by Theorem $4.3, I / I_{w}$ is also an inner function. Therefore, we infer that 
$I=I_{w}$ (modulo constants). This contradiction yields that $I J N^{p}$ does not have the separation property. This completes the proof of the theorem.

\section{Weakly dense ideals in $N^{p}$}

Beurling's invariant subspaces theorem holds for each Hardy space $H^{q}, 0<$ $q<\infty$, the Smirnov class $N^{+}$(see Remark 2), and for all Privalov spaces $N^{p}$ with $1<p<\infty$ (Theorem 2.3). This means that if $E$ is a closed subspace of one of these spaces, denoting by $X$, and if $E$ is invariant under multiplication by $z$, then $E=I X$ for some $X$-inner function $I$.

Since the spaces $N^{p}$ are not locally convex, it is possible that some closed ideals are weakly dense (dense in the weak topology of $N^{p}$ ). In this section we give a construction of such ideals for any $p>1$.

Recall that an inner function $I$ is called a weak outer function in $N^{p}$ if $I N^{p}$ is closed in the weak topology of $N^{p}$; that is, if $\left[I N^{p}\right]_{w}=N^{p}$. This means that $I_{w}=1$, where $I_{w}$ is an inner function as in Theorem 4.3 such that

$$
\left[I N^{p}\right]_{w}=I_{w} N^{p} .
$$

In other words, an inner function $I$ is a weak outer function in $N^{p}$ if and only if the principal ideal $I N^{p}$ is weakly dense. The main result of this paper (Theorem 5.5) gives a large class of positive singular measures $\mu$, depending on $p$, such that associated singular inner functions are weak outer functions in the space $N^{p}$.

By Theorem 3.2, $\left\{\|\cdot\|_{p, c}\right\}_{c>0}$ and $\left\{|\|\cdot\||_{p, c}\right\}_{c>0}$ defined by (3.1) and (3.2), respectively, are equivalent families of seminorms. For simplicity, in this section we write $\|\cdot\|_{c}$ instead of $\|\cdot\|_{p, c}$.

For every $c>0$, we define the function $\|\cdot\|_{c}^{\sim}$ on $F^{p}$ by

$$
\|f\|_{c}^{\sim}=\left(\frac{1}{\pi} \iint_{\mathbb{D}}\left|f\left(r e^{i \theta}\right)\right|^{2} \exp \left(-\frac{c}{(1-r)^{1 / p}}\right) r d r d \theta\right)^{1 / 2}, \quad f \in F^{p} .
$$

It follows easily by Minkowski's inequality that $\|\cdot\|_{c}^{\sim}$ satisfies the triangle inequality for any $c>0$, and hence $\|\cdot\| \tilde{c}$ is a norm on $F^{p}$.

Lemma 5.1. Let $p>1$ and $c>0$ be any fixed. Then there exist positive constants $A$ and $B$, depending only on $p$ and $c$, such that

$$
\|f\|_{c}^{\sim} \leq A\|f\|_{c_{1}}, \quad f \in F^{p}
$$

and

$$
\|f\|_{c} \leq B\|f\|_{c_{2}}^{\sim}, \quad f \in F^{p}
$$

where $c_{1}=\frac{c^{p /(p+1)}}{2}$ and $c_{2}=\left(\frac{c^{p+1}}{3 \cdot 6^{p+1}}\right)^{1 / p}$. Hence, the families $\left\{\|\cdot\|_{c}\right\}_{c>0}$ and $\left\{\|\cdot\|_{c}^{\sim}\right\}_{c>0}$ induce the same topology on $F^{p}$. 
Proof. Suppose that $f \in F^{p}, f \not \equiv 0$, with Taylor expansion $f(z)=\sum_{n=0}^{\infty} a_{n} z^{n}$ on $\mathbb{D}$. Given any constant $c>0$ put $c_{2}=\left(\frac{c^{p+1}}{3 \cdot 6^{p+1}}\right)^{1 / p}$. Then by $[21$, the first inequality on p. 146], there is $m \in \mathbb{N}$ so that for all $n \geq m$ holds

$$
\int_{0}^{1} r^{n} \exp \left(-\frac{c_{2}}{(1-r)^{1 / p}}\right) d r \geq \exp \left(-6\left(c_{2}\right)^{p /(p+1)} n^{1 /(p+1)}\right) .
$$

Assuming that $L \leq 1$ is a positive constant such that

$$
\int_{0}^{1} r^{2 n+1} \exp \left(-\frac{c_{2}}{(1-r)^{1 / p}}\right) d r \geq L \exp \left(-6\left(c_{2}\right)^{p /(p+1)}(3 n)^{1 /(p+1)}\right)
$$

for all $n=0,1, \ldots,[m / 2]-1$, then by (5.1) and (5.4) for each $c_{2}>0$ we have

$$
\begin{aligned}
\left(\|f\|_{c_{2}}\right)^{2}= & \frac{1}{\pi} \int_{0}^{2 \pi} \int_{0}^{1} f\left(r e^{i \theta}\right) \overline{f\left(r e^{i \theta}\right)} \exp \left(-\frac{c_{2}}{(1-r)^{1 / p}}\right) r d r d \theta \\
= & \frac{2 \pi}{\pi} \sum_{n=0}^{\infty}\left|a_{n}\right|^{2} \int_{0}^{1} r^{2 n+1} \exp \left(-\frac{c_{2}}{(1-r)^{1 / p}}\right) d r \\
= & 2\left(\sum_{n=0}^{\left[\frac{m}{2}\right]-1}+\sum_{n=\left[\frac{m}{2}\right]}^{\infty}\right) \\
\geq & 2\left(\sum_{n=0}^{L}\left|a_{n}\right|^{2} \exp \left(-6\left(c_{2}\right)^{p /(p+1)}(3 n)^{1 /(p+1)}\right)\right. \\
& \left.+\sum_{n=\left[\frac{m}{2}\right]}^{\infty}\left|a_{n}\right|^{2} \exp \left(-6\left(c_{2}\right)^{p /(p+1)}(2 n+1)^{1 /(p+1)}\right)\right) \\
\geq & 2 L\left(\sum_{n=0}^{\left[\frac{m}{2}\right]-1}\left|a_{n}\right|^{2} \exp \left(-6\left(c_{2}\right)^{p /(p+1)}(3 n)^{1 /(p+1)}\right)\right. \\
& \left.+\sum_{n=\left[\frac{m}{2}\right]}^{\infty}\left|a_{n}\right|^{2} \exp \left(-6\left(c_{2}\right)^{p /(p+1)}(3 n)^{1 /(p+1)}\right)\right) \\
& 2 L \sum_{n=0}^{\infty}\left|a_{n}\right|^{2} \exp \left(-6\left(c_{2}\right)^{p /(p+1)}(3 n)^{1 /(p+1)}\right) .
\end{aligned}
$$

By Cauchy-Schwarz inequality, for positive numbers $x_{n}, y_{n}$, with $n=1,2, \ldots, k$, holds

$$
\left(\sum_{n=1}^{k} x_{n} y_{n}\right)^{2}=\left(\sum_{n=1}^{k}\left(x_{n} \sqrt{y_{n}}\right) \sqrt{y_{n}}\right)^{2} \leq\left(\sum_{n=1}^{k} x_{n}^{2} y_{n}\right)\left(\sum_{n=1}^{k} y_{n}\right) .
$$


The above inequality with $x_{n}=\left|a_{n}\right|$ and $y_{n}=\exp \left(-c n^{1 /(p+1)}\right)$ as $k \rightarrow \infty$ yields

$$
\begin{aligned}
\left(\|f\|_{c}\right)^{2} & =\left(\sum_{n=0}^{\infty}\left|a_{n}\right| \exp \left(-c n^{1 /(p+1)}\right)\right)^{2} \\
& \leq\left(\sum_{n=0}^{\infty}\left|a_{n}\right|^{2} \exp \left(-c n^{1 /(p+1)}\right)\right)\left(\sum_{n=0}^{\infty} \exp \left(-c n^{1 /(p+1)}\right)\right) \\
& =K \sum_{n=0}^{\infty}\left|a_{n}\right|^{2} \exp \left(-c n^{1 /(p+1)}\right) \\
& =K \sum_{n=0}^{\infty}\left|a_{n}\right|^{2} \exp \left(-6\left(c_{2}\right)^{p /(p+1)}(3 n)^{1 /(p+1)}\right),
\end{aligned}
$$

where $0<K<+\infty$. The inequalities (5.5) and (5.6) immediately yield

$$
\left(\|f\|_{c_{2}}^{\sim}\right)^{2} \geq \frac{2 L}{K}\left(\|f\|_{c}\right)^{2}
$$

On the other hand, we have

$$
\begin{aligned}
\left(\|f\|_{c}^{\sim}\right)^{2} & =\frac{1}{\pi} \int_{0}^{2 \pi} \int_{0}^{1} f\left(r e^{i \theta}\right) \overline{f\left(r e^{i \theta}\right)} \exp \left(-\frac{c}{(1-r)^{1 / p}}\right) r d r d \theta \\
& =2 \sum_{n=0}^{\infty}\left|a_{n}\right|^{2} \int_{0}^{1} r^{2 n+1} \exp \left(-\frac{c}{(1-r)^{1 / p}}\right) d r
\end{aligned}
$$

Since by $[21$, the first inequality on p. 145 with $\beta=1 / p]$, for $n=0,1,2, \ldots$,

$$
r^{n} \exp \left(-\frac{c}{(1-r)^{1 / p}}\right) \leq \exp \left(-c^{p /(p+1)} n^{1 /(p+1)}\right), \quad 0<r<1,
$$

it follows that

$$
\begin{aligned}
\left(\|f\|_{c}^{\sim}\right)^{2} & \leq 2\left(\sum_{n=0}^{\infty}\left|a_{n}\right| \exp \left(-\frac{c^{p /(p+1)}}{2} n^{1 /(p+1)}\right)\right)^{2} \\
& =2\left(\|f\|_{c_{1}}\right)^{2}
\end{aligned}
$$

with $c_{1}=\left(c^{p /(p+1)}\right) / 2$. By setting $A=\sqrt{2}$, the above inequality yields (5.2). This concludes the proof.

Recall that the modulus of continuity $\omega_{\mu}$ of a finite Borel measure $\mu$ on the unit circle $\mathbb{T}$ is defined by

$$
\omega_{\mu}(t)=\sup _{|L| \leq t} \mu(L) \quad(t>0),
$$

where the supremum is taken on every subarcs $L$ of $\mathbb{T}$ whose normalized Lebesgue measure (lenght) $|L| \leq t$.

Observe that from the condition $\omega_{\mu}(t)=O(t)$ it follows that the measure $\mu$ is absolutely continuous with respect to the normalized Lebesgue measure $|\cdot|$ 
on $\mathbb{T}$. Furthermore, it is known that there are positive singular measures with prescribed modulus of continuity (of higher order than $O(t)$ ); for example, with the modulus of continuity $\omega_{\mu}(t)=O\left(t \log \frac{1}{t}\right)$. It is pointed out in [4] that such a measure can be constructed as the Lebesgue function over a Cantor set with variable ratio of dissection. Another example is based on Riesz products (see [3]) that we use here in order to obtain the following result.

Lemma 5.2. For any positive number $\alpha$ such that $0<\alpha<1$ there exists a positive singular Borel measure $\mu$ on $\mathbb{T}$ with the modulus of continuity

$$
\omega_{\mu}(t)=O\left(t^{\alpha}\right) \quad(t>0) .
$$

Proof. We will construct a continuous nondecreasing function $F$ on $[0,2 \pi]$ which generates measure $\mu$ such that

$$
\mu([x, y])=F(x)-F(y)
$$

for any segment $[x, y]$ with $0 \leq y \leq x \leq 2 \pi$, and $\omega_{\mu}(t)$ satisfies (5.7). Recall that the modulus of continuity $\omega(t ; F)$ of a continuous complex-valued function $F$ defined on $[0,2 \pi]$ is given by

$$
\omega(t ; F)=\sup _{|x-y| \leq t}|F(x)-F(y)| .
$$

Moreover, $F$ belongs to the Lipschitz class $\Lambda_{\alpha}$ with $0<\alpha \leq 1$ if $\omega(t ; F)=O\left(t^{\alpha}\right)$ as $t \rightarrow 0$.

Given $0<\alpha<1$, consider a constant sequence $\left\{a_{j}\right\}_{j}$ with $a_{j}=1$ for all $j=1,2, \ldots$, and a sequence $\left\{n_{j}\right\}_{j}$ with $n_{j}=q^{j}$ for all $j=1,2, \ldots$, where $q>3$ is a positive integer for which $q^{1-\alpha} \geq 2$. Now define a sequence $\left\{p_{k}(t)\right\}_{k}$ of trigonometric polynomials as a Riesz product

$$
\begin{aligned}
p_{k}(t) & =\prod_{j=1}^{k}\left(1+a_{j} \cos n_{j} t\right) \\
& =1+\sum_{i=1}^{m_{k}} c_{i} \cos i t, \quad k=1,2, \ldots,
\end{aligned}
$$

with $m_{k}=n_{1}+\cdots+n_{k}=\frac{q\left(1-q^{k}\right)}{1-q}$ and suitable coefficients $c_{i}$. Since $n_{j+1} / n_{j}=$ $q>3$ for all $j=1,2, \ldots$, as noticed in [3, pp. 1264-1265], it follows by [18] and $[27$, p. 208] that

$$
F(x):=\lim _{k \rightarrow \infty} \int_{0}^{x} p_{k}(t) d t
$$

exists for all $x, 0 \leq x \leq 2 \pi$, and $F$ is a continuous nondecreasing function on $[0,2 \pi]$. We say that the function $F$ is generated by a Riesz product. Further, we have

$$
\prod_{j=1}^{k}\left(1+\left|a_{j}\right|\right)=2^{k} \leq q^{(1-\alpha) k}=\left(n_{k}\right)^{1-\alpha},
$$


and hence sequences $\left\{a_{j}\right\}_{j}$ and $\left\{n_{j}\right\}_{j}$ satisfy the asymptotic condition (6) from [3, Theorem 1]. Then by the same theorem from [3], the function $F$ belongs to the Lipschitz class $\Lambda_{\alpha}$, and so for a measure $\mu$ defined by (5.8) we obtain

$$
\begin{aligned}
\omega_{\mu}(t) & =\omega(t ; F) \\
& =\sup _{|x-y| \leq t}|F(x)-F(y)| \\
& =O\left(t^{\alpha}\right) .
\end{aligned}
$$

Finally, since $\sum_{j=1}^{\infty} a_{j}^{2}=\infty$, by [3, Theorem A], we conclude that $F$ is a singular function. Consequently, $\mu$ is a singular Borel measure on $\mathbb{T}$ whose modulus of continuity satisfies (5.7). This completes the proof.

We will need the following result in the proof of the main result.

Lemma 5.3. Let

$$
S_{\mu}(z)=\exp \left(-\int_{0}^{2 \pi} H\left(z, e^{i t}\right) d \mu(t)\right)
$$

be a singular inner function, where $H\left(z, e^{i t}\right)=\left(e^{i t}+z\right)\left(e^{i t}-z\right)^{-1}$, and let $\mu$ be a positive singular Borel measure with the modulus of continuity $\omega_{\mu}(t)=$ $O\left(t^{(p-1) / p}\right)$. Then

$$
\left|S_{\mu}\left(r e^{i \theta}\right)\right| \geq \exp \left(-\frac{C}{(1-r)^{1 / p}}\right), \quad 0 \leq r<1,
$$

for some constant $C>0$.

Proof. Obviously,

$$
-\log \left|S_{\mu}\left(r e^{i \theta}\right)\right|=\int_{0}^{2 \pi} P(r, \theta-t) d \mu(t),
$$

with the Poisson kernel

$$
P(r, \theta-t)=\Re H\left(z, e^{i t}\right)=\frac{1-r^{2}}{1-2 r \cos (\theta-t)+r^{2}}, \quad z=r e^{i \theta} .
$$

Since $\sin x \geq 2 x / \pi$ for each $0 \leq x \leq \pi / 2$, we have

$$
\begin{aligned}
1-2 r \cos (\theta-t)+r^{2} & =(1-r)^{2}+4 r \sin ^{2} \frac{\theta-t}{2} \\
& \geq(1-r)^{2}+\frac{4 r}{\pi^{2}}(\theta-t)^{2} .
\end{aligned}
$$

As for $r \geq 1 / 17,\left(372 r / \pi^{2}\right)(\theta-t)^{2} \geq 2(\theta-t)^{2}$, and since $|\theta-t| \leq 2 \pi$, the inequality $91(1-r)^{2}>2(\theta-t)^{2}$ holds for $r<1 / 17$, in both cases it is obvious that

$$
2\left((1-r)^{2}+(\theta-t)^{2}\right) \leq 93\left((1-r)^{2}+\frac{4 r}{\pi^{2}}(\theta-t)^{2}\right)
$$


Now from (5.11)-(5.13) we immediately obtain

$$
\begin{aligned}
P(r, \theta-t) & \leq \frac{2(1-r)}{1-2 r \cos (\theta-t)+r^{2}} \\
& \leq \frac{93(1-r)}{(1-r)^{2}+(\theta-t)^{2}}, \quad z=r e^{i \theta} .
\end{aligned}
$$

If we put $\delta=2 \pi / n$, then by (5.10), (5.11) and (5.14) and the assumption $\omega_{\mu}(\delta)=O\left(\delta^{(p-1) / p}\right)$ of the lemma, we obtain

$$
\begin{aligned}
-\log \left|S_{\mu}\left(r e^{i \theta}\right)\right| & \leq c \delta^{\frac{p-1}{p}} \sum_{k=0}^{n-1} \max _{k \delta \leq t \leq(k+1) \delta}\left\{\frac{1-r}{(1-r)^{2}+(\theta-t)^{2}}\right\} \\
& \leq c \delta^{\frac{p-1}{p}}\left(\frac{1}{1-r}+\sum_{k=1}^{n-1} \frac{1-r}{(1-r)^{2}+k^{2} \delta^{2}}\right)
\end{aligned}
$$

for a positive constant $c$. Then assuming $n \in \mathbb{N}$ such that $1 /(1-r) \leq n<$ $1+1 /(1-r)$ for such $n$, we have $2 \pi(1-r) /(2-r)<\delta \leq 2 \pi(1-r)$, and from the inequality (5.15) we obtain

$$
\begin{aligned}
-\log \left|S_{\mu}\left(r e^{i \theta}\right)\right| & \leq c \delta^{\frac{p-1}{p}}\left(\frac{1}{1-r}+\sum_{k=1}^{n-1} \frac{1}{1-r} \cdot \frac{1}{1+\pi^{2} k^{2}}\right) \\
& \leq c(2 \pi)^{\frac{p-1}{p}}(1-r)^{\frac{p-1}{p}}\left(\frac{1}{1-r}+\frac{1}{(1-r) \pi^{2}} \sum_{k=1}^{\infty} \frac{1}{k^{2}}\right) \\
& =c(2 \pi)^{\frac{p-1}{p}}(1-r)^{\frac{p-1}{p}} \cdot \frac{7}{6(1-r)} \\
& =C(1-r)^{-\frac{1}{p}}
\end{aligned}
$$

for a constant $C=\left(7 c(2 \pi)^{(p-1) / p}\right) / 6$. Hence we have

$$
\left|S_{\mu}\left(r e^{i \theta}\right)\right| \geq \exp \left(-\frac{C}{(1-r)^{1 / p}}\right) .
$$

This is the desired estimate (5.9).

Lemma 5.4. For any $c>0$ denote by $[E]_{c}$ the closure of a subspace $E$ of $N^{p}$ in the normed space $\left(F^{p},\|\cdot\|_{c}^{\sim}\right)$. Then for arbitrary inner functions $I$ and $J$

$$
\left[I J N^{p}\right]_{c}=\left[I\left[J N^{p}\right]_{c}\right]_{c} .
$$

In particular, if $\left[J N^{p}\right]_{c}=N^{p}$, then

$$
\left[I J N^{p}\right]_{c}=\left[I N^{p}\right]_{c} .
$$

Proof. Since $J N^{p} \subseteq\left[J N^{p}\right]_{c}$, we have $\left[I J N^{p}\right]_{c} \subseteq\left[I\left[J N^{p}\right]_{c}\right]_{c}$. Obviously, for any function $h \in H^{\infty}$ with the norm $\|h\|_{\infty}=\max _{z \in \mathbb{D}}|h(z)|$ and each $f \in N^{p}$ holds

$$
\|h f\|_{c}^{\sim} \leq\|h\|_{\infty}\|f\|_{c}^{\sim} .
$$


Therefore, for each $f$ and $g$ in $N^{p}$ we obtain

$$
\|I J g-I f\|_{c}^{\sim} \leq\|I\|_{\infty}\|J g-f\|_{c}^{\sim},
$$

and hence $\left[I\left[J N^{p}\right]_{c}\right]_{c} \subseteq\left[I J N^{p}\right]_{c}$. This concludes the proof.

According to Theorem 2.5, the principal ideal in $N^{p}$ is dense in $N^{p}$ if and only if it is generated by an outer function. Although we have been unable to give a complete characterization of weakly dense ideals in $N^{p}$, the following theorem describes a large class of such ideals in $N^{p}$.

Theorem 5.5. Let $\mathcal{M}$ be a closed ideal in $N^{p}$ that is weakly dense in $N^{p}$. Then $\mathcal{M}$ is a principal ideal generated by a singular inner function. Conversely, if $S_{\mu}$ is a non-trivial singular inner function with the associated measure $\mu$ whose modulus of continuity $\omega_{\mu}$ satisfies

$$
\omega_{\mu}(t)=O\left(t^{\frac{p-1}{p}}\right), \quad t>0
$$

then the ideal $S_{\mu} N^{p}$ is weakly dense in $N^{p}$.

Proof. Let $\mathcal{M}$ be a closed ideal with respect to the metric topology $\rho_{p}$ of $N^{p}$ that is weakly dense in $N^{p}$. Then by Theorem 2.1, there is a unique (modulo constants) inner function $I$ such that $\mathcal{M}=I N^{p}$. Suppose that a function $I$ vanishes in $\mathbb{D}$, i.e., $I(z)=B(z) S_{\mu}(z)$, where $B$ is a nontrivial Blaschke factor of $I$, and $S_{\mu}$ is a singular inner factor of $I$. Let $\xi$ be an arbitrary zero of $B$. Since by Theorem 3.8, the evaluation functional $\delta_{\xi}$ defined as $\delta_{\xi}(f)=f(\xi), f \in N^{p}$, is continuous, the assumption that $\mathcal{M}$ is weakly dense implies that there is a sequence $\left\{f_{n}\right\}_{n} \subset N^{p}$ such that $\delta_{\xi}\left(B S_{\mu} f_{n}\right) \rightarrow \delta_{\xi}(1)$ as $n \rightarrow \infty$. This means that $B(\xi) S_{\mu}(\xi) f_{n}(\xi) \rightarrow 1$ as $n \rightarrow \infty$, what is impossible in view of the fact that $B(\xi)=0$. This contradiction shows that $I$ is a singular inner function.

Conversely, suppose that $S_{\mu}$ is a singular inner function with the associated measure $\mu$ whose modulus of continuity $\omega_{\mu}(t)=O\left(t^{(p-1) / p}\right)$. By Theorem 3.3, $F^{p}$ is a Fréchet algebra in which $f_{r} \rightarrow f$ as $r \rightarrow 1$ for each $f \in N^{p}$ with $f_{r}(z)=f(r z), z \in \mathbb{D}$. Since each function $f_{r}$ can be uniformly approximated on the closed disk $\overline{\mathbb{D}}:|z| \leq 1$ by partial sums of its Taylor expansion, it follows that the space $\mathcal{P}$ of polynomials is dense in $F^{p}$. Hence, the density of $S_{\mu} N^{p}$ in $F^{p}$ is equivalent to the fact that the set $\mathcal{P} S_{\mu}=\left\{P S_{\mu}: P \in \mathcal{P}\right\}$ is dense in $F^{p}$. Since by Lemma 5.1 , families of seminorms $\left\{\|\cdot\|_{c}^{\sim}\right\}_{c>0}$ and $\left\{\|\cdot\|_{c}\right\}_{c>0}$ given by (5.1) and (3.1), respectively, define the same topology on $F^{p}$, it follows from (i) $\Leftrightarrow$ (ii) of Corollary 4.7 that the ideal $S_{\mu} N^{p}$ is weakly dense in $N^{p}$ if and only if it is dense in the normed spaces $\left(F^{p},\|\cdot\|_{c} \tilde{c}\right)_{c>0}$ for each $c>0$.

By (5.9) of Lemma 5.3, there is a constant $C>0$ such that for the minimum modulus $m(r)=\min _{|z|=r}\left|S_{\mu}(z)\right|$ of $S_{\mu}$ holds

$$
m(r) \geq \exp \left(-\frac{C}{(1-r)^{1 / p}}\right) \quad \text { for } \quad 0 \leq r<1 .
$$

Denote by $p_{n} n$ 'th Cesàrov's sum (n'th arithmetic mean of the partial sums of the Taylor expansion) of the function $1 / S_{\mu}(z)$. Then $p_{n}(z) \rightarrow 1 / S_{\mu}(z)$ 
uniformly on compact subsets of $\mathbb{D}$, and hence $p_{n}(z) S_{\mu}(z) \rightarrow 1$ for each $z \in \mathbb{D}$. By [11, Kap.1, Satz 1, p. 22] there holds

$$
\max _{|z|=r}\left|p_{n}(z)\right| \leq \max _{|z|=r} \frac{1}{\left|S_{\mu}(z)\right|},
$$

whence by (5.17) immediately follows

$$
\max _{|z|=r}\left|p_{n}(z)\right| \leq \frac{1}{m(r)} \leq \exp \left(\frac{C}{(1-|z|)^{1 / p}}\right) \text { for } 0 \leq|z|=r<1 .
$$

Since $\left|S_{\mu}(z)\right|<1$ for each $z \in \mathbb{D}$, combining (5.18) and the inequality $|1+v|^{2} \leq$ $2\left(1+|v|^{2}\right)$, for any positive constant $c$ and $0 \leq|z|<1$ we obtain

$$
\begin{aligned}
& \left|1-p_{n}(z) S_{\mu}(z)\right|^{2} \exp \left(-\frac{c}{(1-|z|)^{1 / p}}\right) \\
\leq & 2\left|p_{n}(z)\right|^{2} \exp \left(-\frac{c}{(1-|z|)^{1 / p}}\right)+2 \exp \left(-\frac{c}{(1-|z|)^{1 / p}}\right) \\
\leq & 2 \exp \left(-\frac{c-C}{2(1-|z|)^{1 / p}}\right)+2 \exp \left(-\frac{c}{(1-|z|)^{1 / p}}\right) .
\end{aligned}
$$

It follows from the above inequality that for a constant $c$ such that $c \geq C$, a sequence $\left\{f_{n}\right\}_{n}$ of functions defined as

$$
f_{n}(z)=\left|1-p_{n}(z) S_{\mu}(z)\right|^{2} \exp \left(-\frac{c}{(1-|z|)^{1 / p}}\right), \quad z \in \mathbb{D}, n \in \mathbb{N},
$$

is bounded by modulus by a function which is integrable on $\mathbb{D}$ with respect to the area normalized Lebesgue measure $r d r d \theta / \pi$. Since $p_{n}(z) S_{\mu}(z) \rightarrow 1$ as $n \rightarrow \infty$ for each $z \in \mathbb{D}$, it follows by the Dominated Convergence Theorem that for any fixed $c \geq C$ a sequence $\left\{\left(\left\|p_{n} S_{\mu}-1\right\|_{c}^{\sim}\right)^{2}\right\}_{n}$ defined as

$$
\begin{aligned}
& \left(\left\|p_{n} S_{\mu}-1\right\|_{c}^{\sim}\right)_{n}^{2} \\
= & \frac{1}{\pi} \int_{0}^{2 \pi} \int_{0}^{1}\left|1-p_{n}\left(r e^{i \theta}\right) S_{\mu}\left(r e^{i \theta}\right)\right|^{2} \exp \left(-\frac{c}{\left(1-\left|r e^{i \theta}\right|\right)^{1 / p}}\right) r d r d \theta, \quad n \in \mathbb{N},
\end{aligned}
$$

converges to zero as $n \rightarrow \infty$. Hence, the space $S_{\mu} \mathcal{P}$ is dense in $\left(F^{p},\|\cdot\|_{c}\right)$ for each $c \geq C$.

Now suppose that $0<c<C$. Let $n$ be a positive integer such that $1 / n<$ $c / C$. Then applying the above argument to the singular inner function $\left(S_{\mu}\right)^{1 / n}$, we conclude that the space $\left(S_{\mu}\right)^{1 / n} \mathcal{P}$ is dense in $\left(F^{p},\|\cdot\|_{c}\right)$, i.e., according to the notation from Lemma 5.4 , we have $\left[\left(S_{\mu}\right)^{1 / n} N^{p}\right]_{c}=F^{p}$. Therefore, by (5.16) of Lemma 5.4, we have

$$
\begin{aligned}
{\left[\left(S_{\mu}\right)^{2 / n} N^{p}\right]_{c} } & =\left[\left(S_{\mu}\right)^{1 / n}\left[\left(S_{\mu}\right)^{1 / n} N^{p}\right]_{c}\right]_{c} \\
& =\left[\left(S_{\mu}\right)^{1 / n} F^{p}\right]_{c} \\
& =F^{p} .
\end{aligned}
$$


Hence, repeating the application of (5.19) n-1 times, we obtain $\left[S_{\mu} N^{p}\right]_{c}=F^{p}$. Therefore, $S_{\mu} N^{p}$ is dense in the space $\left(F^{p},\left\{\|\cdot\|_{c}^{\sim}\right\}\right)$ for each $c>0$. This completes the proof.

Recall that an inner function $I$ is said to be a weak outer function in $N^{p}$ if the set $I N^{p}:=\left\{I f: f \in N^{p}\right\}$ is dense in the weak topology of $N^{p}$. Then the second assertion of Theorem 5.5 can be formulated as follows.

Corollary 5.6. Every non-trivial singular inner function $S_{\mu}$ with the associated measure $\mu$ whose modulus of continuity $\omega_{\mu}(t)=O\left(t^{(p-1) / p}\right)$ is a weak outer function in $N^{p}$.

For a closed subspace $E$ of $N^{p}$, and for any $f \in N^{p}$, let $\bar{f}$ denote the coset of $f+N^{p}$ in the quotient space $N^{p} / E$. Define

$$
\|\bar{f}\|=\inf \{\|g\|: g \in \bar{f}\}, \quad f \in N^{p}
$$

where $\|g\|=\rho_{p}(g, 0)$. Then the function $\bar{\rho}_{p}$ defined as

$$
\bar{\rho}_{p}(\bar{f}, \bar{g})=\|\bar{f}-\bar{g}\|, \quad \bar{f}, \bar{g} \in N^{p} / E,
$$

is an additively invariant metric that induces the quotient topology on $N^{p} / E$. It is well known that $N^{p} / E$ is an $F$-space with respect to the metric $\bar{\rho}_{p}$ (cf. [22, Theorem 12.3.5, p. 264]).

Corollary 5.7. Let $S_{\mu}$ be a singular inner function described in Theorem 5.5. Then the following statements about the closed ideal $S_{\mu} N^{p}$ in $N^{p}$ hold.

(i) If $\phi$ is a continuous linear functional on $N^{p}$ which annihilates $S_{\mu} N^{p}$, then $\phi$ is the zero functional.

(ii) The quotient space $\left(N^{p} / S_{\mu} N^{p}, \bar{\rho}_{p}\right)$ is an $F$-space with the trivial dual.

(iii) The space $S_{\mu} N^{p}$ does not have the separation property, and hence does not have the Hahn-Banach property.

Proof. Properties (i) and (ii) immediately follow from the fact that by Theorem 5.5 , the ideal $S_{\mu} N^{p}$ is weakly dense in $N^{p}$ (cf. [4, Theorem 16, p. 59], where the analogous assertions are given for any topological vector space with enough continuous linear functionals to separate points). Since $\left[S_{\mu} N^{p}\right]_{w}=N^{p} \neq$ $S_{\mu} N^{p}$, we see that $S_{\mu} N^{p}$ is not weakly closed, and by Lemma $4.1, S_{\mu} N^{p}$ does not have the separation property.

\section{References}

[1] J. S. Choa and H. O. Kim, Composition operators on some F-algebras of holomorphic functions, Nihonkai Math. J. 7 (1996), no. 1, 29-39.

[2] P. L. Duren, Theory of $H^{p}$ Spaces, Academic Press, New York, 1970.

[3] _ Smootheness of functions generated by Riesz products, Proc. Amer. Math. Soc. 16 (1965), 1263-1268.

[4] P. L. Duren, B. W. Romberg, and A. L. Shields, Linear functionals on $H^{p}$ spaces with $0<p<1$, J. Reine Angew. Math. 238 (1969), 32-60.

[5] C. M. Eoff, Fréchet envelopes of certain algebras of analytic functions, Michigan Math. J. 35 (1988), no. 3, 413-426. 
[6] _ A representation of $N_{\alpha}^{+}$as a union of weighted Hardy spaces, Complex Variables Theory Appl. 23 (1993), no. 3-4, 189-199.

[7] J. Garnett, Bounded Analytic Functions, Academic Press, New York, 1981.

[8] H. Helson, Lectures on Invariant Subspaces, Academic Press, New York, 1964.

[9] K. Hoffman, Banach Spaces of Analytic functions, Prentice-Hall, Englewood Cliffs, NJ, 1962.

[10] J. L. Kelley, I. Namioka et al., Linear Topological Spaces, D. Van Nostrand Co., Inc., Princeton, N.J. 1963.

[11] E. Landau, Darstellung und Begrundung einiger neurer Ergebnisse der Funktiontheorie, Springer, Berlin, 1929.

[12] Y. Matsugu, Invariant subspaces of the Privalov spaces, Far East J. Math. Sci. (FJMS) 2 (2000), no. 4, 633-643.

[13] R. Meštrović and Ž. Pavićević, The logarithmic analogue of Szegö's theorem, Acta Sci. Math. (Szeged) 64 (1998), no. 1-2, 97-102.

[14] R. Meštrović and A. V. Subbotin, Multipliers and linear functionals of I. I. Privalov spaces of functions holomorphic in the disk, Dokl. Akad. Nauk 365 (1999), no. 4, 452454.

[15] J. E. McCarthy, Topologies on the Smirnov class, J. Funct. Anal. 104 (1992), no. 1, $229-241$.

[16] N. Mochizuki, Algebras of holomorphic functions between $H^{p}$ and $N_{*}$, Proc. Amer. Math. Soc. 105 (1989), no. 4, 898-902.

[17] I. I. Privalov, Boundary properties of Analytic Functions, Moscow University Press, Moscow, 1950.

[18] F. Riesz, Über die Fourierkoeffizienten einer stetigen Funktion von beschränkter Schwankung, Math. Z. 2 (1918), 312-315.

[19] J. W. Roberts and M. Stoll, Prime and principal ideals in the algebra $N^{+}$, Arch. Math. (Basel) 27 (1976), no. 4, 387-393; Correction, ibid 30 (1978), 672.

[20] J. H. Shapiro, Mackey topologies, reproducing kernels, and diagonal maps on the Hardy and Bergman spaces, Duke Math. J. 43 (1976), no. 1, 187-202.

[21] M. Stoll, Mean growth and Taylor coefficients of some topological algebras of analytic functions, Ann. Polon. Math. 35 (1977/78), no. 2, 139-158.

[22] A. Wilansky, Topology for Analysis, Ginn and Co., Waltham, 1970.

[23] N. Yanagihara, Multipliers and linear functionals for the class $N^{+}$, Trans. Amer. Math. Soc. 180 (1973), 449-461.

[24] - The containing Fréchet space for the class $N^{+}$, Duke Math. J. 40 (1973), 93-103.

[25] A. I. Zayed, Topological vector spaces of analytic functions, Complex Variables Theory Appl. 2 (1983), no. 1, 27-50.

[26] _ Recoverability of some classes of analytic functions from their boundary values, Proc. Amer. Math. Soc. 87 (1983), no. 3, 493-498.

[27] A. Zygmund, Trigonometric Series. 2nd ed. Vols. I, II, Cambridge University Press, New York 1959.

RoMEO MEŠTrOviĆ

Department of Mathematics

MARITIME FACULTY

University of Montenegro

Dobrota 36, 85330 Kotor, Montenegro

E-mail address: romeo@ac.me 
ŽARKO PAVIĆEVIĆ

Department of Mathematics

FACUlty of SCIEnCE

UNIVERSity OF MONTENEGRO

DŽordža VAšingtona BB, 81000 PodgoricA, Montenegro

E-mail address: zarkop@ac.me 